\title{
Cancer Survivorship Care Roles for Primary Care Physicians
}

Benjamin F. Crabtree, $P b D, M A^{1,2}$

William L. Miller, MD, $M A^{3}$

Jenna Howard, $P b D^{1}$

Ellen B. Rubinstein, $P b D, M A^{4}$

Jennifer Tsui, $\mathrm{PbD}, \mathrm{MPH}$

Shawna V. Hudson, $P b D^{1,2}$

Denalee O'Malley, PbD, MSW $W^{1,2}$

Jeanne M. Ferrante, MD, MPH $H^{1,2}$

Kurt C. Stange, $M D, P b D^{5}$

'Rutgers Robert Wood Johnson Medical School, New Brunswick, New Jersey

${ }^{2}$ Rutgers Cancer Institute of New Jersey, New Brunswick, New Jersey

${ }^{3}$ Lehigh Valley Health Network, Allentown, Pennsylvania

${ }^{4}$ North Dakota State University, Fargo, North Dakota

${ }^{5}$ Case Western Reserve University, Cleveland, Ohio

Conflicts of interest: authors report none.

\section{CORRESPONDING AUTHOR}

Benjamin F. Crabtree, PhD, MA Rutgers Robert Wood Johnson Medical School

112 Paterson Street, \#413

New Brunswick, NJ 08901

benjamin.crabtree@rutgers.edu

\begin{abstract}
PURPOSE Despite a burgeoning population of cancer survivors and pending shortages of oncology services, clear definitions and systematic approaches for engaging primary care in cancer survivorship are lacking. We sought to understand how primary care clinicians perceive their role in delivering care to cancer survivors.
\end{abstract}

METHODS We conducted digitally recorded interviews with 38 clinicians in 14 primary care practices that had national reputations as workforce innovators. Interviews took place during intense case study data collection and explored clinicians' perspectives regarding their role in cancer survivorship care. We analyzed verbatim transcripts using an inductive and iterative immersion-crystallization process.

RESULTS Divergent views exist regarding primary care's role in cancer survivor care with a lack of coherence about the concept of survivorship. A few clinicians believed any follow-up care after acute cancer treatment was oncology's responsibility; however, most felt cancer survivor care was within their purview. Some primary care clinicians considered cancer survivors as a distinct population; others felt cancer survivors were like any other patient with a chronic disease. In further interpretative analysis, we discovered a deeply ingrained philosophy of whole-person care that creates a professional identity dilemma for primary care clinicians when faced with rapidly changing specialized knowledge.

CONCLUSIONS This study exposes an emerging identity crisis for primary care that goes beyond cancer survivorship care. Facilitated national conversations might help specialists and primary care develop knowledge translation platforms to support the prioritizing, integrating, and personalizing functions of primary care for patients with highly complicated issues requiring specialized knowledge.

Ann Fam Med 2020;18:202-209. https://doi.org/10.1370/afm.2498.

\section{INTRODUCTION}

$\mathrm{T}$ he population of adult cancer survivors in the United States is poised to reach 26.1 million by $2040,{ }^{1}$ including large numbers of survivors of childhood cancers who are living longer with co-morbidities that increase with age. ${ }^{2}$ Even as the number of cancer survivors grows, there looms an impending shortage of oncology services to care for them. ${ }^{3-6}$ Over a decade ago, the Institute of Medicine (IOM) released its landmark report charting a path for cancer survivorship by transitioning patients back to primary care. ${ }^{7}$ In 2015 , a commission composed of primary care, oncology, and public health experts described the need to optimize models for primary care integration across the cancer control continuum. ${ }^{8}$ It was hoped that clinical guidelines, ${ }^{9-12}$ and the development of cancer survivorship care plans, ${ }^{7,13}$ could provide guidance to primary care clinicians. Nevertheless, it remains unclear how follow-up needs of survivors should be prioritized and/or integrated into primary care. ${ }^{14}$

There is no clear consensus on the definitions of survivors and survivorship. ${ }^{15-17}$ The National Cancer Institute defines cancer survivors as persons from the time of cancer diagnosis until the end of life, ${ }_{1}^{18}$ while other organizations, such as the American Society of Clinical Oncology, view cancer survivorship care as beginning in "the period following acute diag- 
nosis and treatment." ${ }^{\prime \prime 9}$ Additionally, the IOM report and others consider longer-term survivors, those in the post-treatment period, as persons with cancer who have survived beyond 5 years. ${ }^{7}$ This lack of clear definition creates a nebulous situation as to when primary care should resume responsibility for these patients.

In earlier work, we found little systematic care provided to cancer survivors despite the availability of practice-level resources. ${ }^{20,21}$ Even in practices with Level 3 National Committee of Quality Assurance (NCQA) patient-centered medical home $(\mathrm{PCMH})$ recognition, practice-level awareness and processes for delivering and coordinating cancer survivorship care are absent. ${ }^{20}$ Furthermore, data remain limited on how primary care clinicians perceive their role in delivering care to cancer survivors. ${ }^{22}$ Our own prior investigation suggested possible disagreement among primary care clinicians concerning this role. ${ }^{20}$ Therefore, the goal of this study is to examine individual primary care clinicians' attitudes and beliefs about their role in caring for cancer survivors in practices nationally recognized as workforce innovators.

\section{METHODS}

We analyzed semistructured interviews with 38 clinicians, completed as part of comparative case studies of 14 primary care practices across the United States that were nationally recognized as workforce innovators. ${ }^{20}$ After studying primary care practices and the PCMH intensely over the past 2 decades, ${ }^{23-26}$ several of the authors came into this study with skepticism that a $\mathrm{PCMH}$ would pay particular attention to cancer survivors. Nevertheless, the study team anticipated these practices would have varying intensity of care delivery for cancer survivors.

The Rutgers University Institutional Review Board approved this study, and all clinicians provided informed consent.

\section{Study Sample Selection and Data Collection}

The selection of primary care practices and case study data collection are described elsewhere. ${ }^{20,21}$ Briefly, an executive steering committee ranked a list of 151 practices recognized as workforce innovators that had been compiled for the Robert Wood Johnson Foundation. ${ }^{27}$ We iteratively recruited practices in 3 waves to maximize diversity of practice setting until it became apparent we were not identifying any new information on cancer survivorship. Overall, 20 practices were contacted, 3 declined and 3 expressed interest but were later unresponsive, giving us a final sample of 14 practices.

From 2015-2017, an experienced field researcher visited each practice for 10-12 days, taking observational fieldnotes and conducting key informant interviews with clinicians and staff. We purposely and iteratively selected 38 individual clinicians (medical doctors/doctors of osteopathic medicine [MDs/DOs] and nurse practitioners/physician assistants [NPs/ PAs]) for semistructured, digitally recorded interviews generally after the researcher had been in the practice for a full week. Those interviewed were fully aware of our focus on care of cancer survivors, with respondents asked to describe how they viewed their role in cancer survivorship, decisions of when and where to refer patients, and knowledge about new primary carefriendly survivorship care guidelines. Respondents reflected on their experiences caring for patients with a history of cancer, with interviews ranging from 1-3 hours in length. We imported transcripts (578 pages) into Atlas.ti Version 7.5.13 (Atlas.ti Scientific Software Development $\mathrm{GmbH}$ ) for data management.

\section{Data Analysis}

We used an inductive, iterative process for collaboratively identifying patterns in the data. ${ }^{28}$ All text related to cancer survivor care were extracted through keyword searching ("survivor" and/or "survivorship"). Three authors (B.F.C., J.H., E.B.R.) independently read the resulting text and noted impressions and patterns. They developed an initial codebook based on patterns with the most resonance and then refined code definitions by coding a portion of data together. One author (J.H.) then coded the remaining text. These 3 authors met again to identify and discuss the richest codes. Two authors (J.H., E.B.R.) read these conceptually rich coded data and were struck by the lack of consistency in clinicians' perspectives about primary care's role in cancer survivorship. Returning to the uncoded transcripts, they independently reread all interviews, including those not included in the original data subset. They each created a data matrix that included clinicians' "cancer survivorship perspective" with illustrative quotes and met to compare matrices and resolve inconsistencies. The larger research team reviewed these analyses for a final interpretation of key themes.

\section{RESULTS}

The majority of the sample were physicians $(76 \%)$, female $(60 \%)$, and ranged in age from 20 to 70 years. Most practices were located in suburban areas (57\%). Nine of the 14 practices (64\%) had NCQA Level 3 PCMH status; others included a Veterans Administration practice, a nurse-led Federally Qualified Health Center, and 3 Direct Care Practices (Table 1).

We discovered divergent views on the role of primary care in care of cancer survivors, and a corresponding diversity of approaches, as described below. 
Divergent Views on the Purview of Primary Care in Cancer Survivorship

A minority of clinicians $(\mathrm{n}=5)$ felt providing care to cancer survivors was not within the purview of primary care. They believed any follow-up care after acute treatment was, and should continue to be, oncology's responsibility. As one physician said:

$[\mathrm{M}] \mathrm{y}$ presumption is that if [patients with a history of cancer] have needs, they're going to be hooked in with an oncologist. So, there's a territory or turf issue here. [P13-MD1]

Similarly, a nurse practitioner observed that delivering care to cancer survivors "doesn't come to our attention that much" [P07-NP2] and assumed survivorship "gets taken care of" by oncology. She believed that providing care for cancer survivors was beyond the scope of a typical primary care practice; and that if her practice were to incorporate cancer survivorship care, she said that it would require deciding "that we were going to be some kind of a specialty primary care that made that [cancer survivorship] as our thing."

The majority of clinicians believed cancer survivorship fell within the purview of primary care; however, many described obstacles to providing survivorship care. For example, some clinicians did not feel adequately educated about the needs of cancer survivors. One physician explained:

[Cancer treatment] kind of happens like in a black box... I feel a little intimidated by it, to be honest with you... I don't feel like I know enough about the long-term side effects of the chemo toxic agents [P02-MD2]

Another physician said, "[I]t's hard work to stay current on [cancer treatment], and it's something that I think most of us [in primary care] have low confidence on." [P03-MD1]

Other clinicians highlighted an uneasy relationship with oncology as the major obstacle. One physician's view was "if someone is 10,12, 20 years out [from acute cancer treatment]... we [in primary care] really should be the ones following them." His experience,
Table 1. Practice Descriptions and Characteristics of Clinicians Interviewed in Each

\begin{tabular}{lccccc}
$\begin{array}{l}\text { Practice and } \\
\text { Clinician ID }\end{array}$ & $\begin{array}{c}\text { Age } \\
\text { Range, Y }\end{array}$ & Sex & $\begin{array}{c}\text { Degreel } \\
\text { Specialty }\end{array}$ & $\begin{array}{c}\text { Leadership } \\
\text { Role (Y/N) }\end{array}$ & $\begin{array}{c}\text { Years at } \\
\text { Practice }\end{array}$ \\
\hline Practice 01: Large, ${ }^{a}$ suburban, independent, & physician-owned; NCQA Level 3 & \\
P01-MD1 & $51-60$ & $\mathrm{M}$ & $\mathrm{MD} / \mathrm{IM}$ & $\mathrm{Y}$ & $11-20$ \\
P01-MD2 & $41-50$ & $\mathrm{~F}$ & $\mathrm{MD} / \mathrm{IM}$ & $\mathrm{Y}$ & $11-20$ \\
P01-MD3 & $41-50$ & $\mathrm{~F}$ & $\mathrm{MD} / \mathrm{IM}$ & $\mathrm{Y}$ & $11-20$ \\
P01-PA1 & $21-30$ & $\mathrm{~F}$ & $\mathrm{PA}$ & $\mathrm{N}$ & $1-5$
\end{tabular}

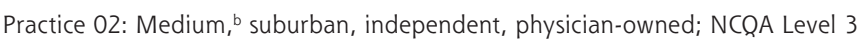

$\begin{array}{lccccc}\text { P02-MD1 } & 41-50 & \mathrm{M} & \text { MD/FM } & \mathrm{Y} & 21+ \\ \text { P02-MD2 } & 41-50 & \mathrm{~F} & \text { MD/FM } & \mathrm{Y} & 11-20\end{array}$

Practice 03: Medium, ${ }^{\text {b }}$ suburban, hospital health system-owned; NCQA Level 3

$\begin{array}{llllll}\text { P03-MD1 } & 31-40 & \mathrm{~F} & \text { MD/FM } & \mathrm{Y} & 6-10 \\ \text { P03-MD2 } & 51-60 & \mathrm{~F} & \text { MD/FM } & \mathrm{N} & 21+ \\ \text { P03-MD3 } & 61-70 & \mathrm{~F} & \text { MD/FM } & \mathrm{N} & 21+\end{array}$

Practice 04: Medium, ${ }^{\mathrm{b}}$ rural, FQHC; NCQA Level 3

$\begin{array}{lccccc}\text { P04-MD1 } & 51-60 & \text { M } & \text { MD/FM } & \text { Y } & 21+ \\ \text { P04-MD2 } & 51-60 & \text { M } & \text { MD/FM } & \text { Y } & 21+ \\ \text { P04-PA1 } & 21-30 & \text { F } & \text { PA } & \text { N } & 1-5\end{array}$

Practice 05: Small, ${ }^{c}$ small city, health system-owned; NCQA Level 3

$\begin{array}{llllll}\text { P05-MD1 } & 71-80 & M & M D / F M & Y & 21+\end{array}$

$\begin{array}{lllll}\text { P05-MD2 } & 61-70 & M & \text { MD/FM } & Y\end{array}$

$\begin{array}{llllll}\text { P05-NP1 } & 61-70 & \text { F } & \text { NP } & \text { N } & 21+\end{array}$

Practice 06: Medium, ${ }^{\mathrm{b}}$ rural, health system-owned FQHC; NCQA Level 3

$\begin{array}{llllll}\text { P06-MD1 } & 41-50 & M & \text { MD/FM } & \text { Y }\end{array}$

$\begin{array}{lllll}\text { P06-MD2 } & 51-60 & F & \text { MD/FM } & \text { N }\end{array}$

$\begin{array}{llllll}\text { P06-MD3 } & 61-70 & \mathrm{~F} & \mathrm{MD} / \mathrm{FM} & \mathrm{N} & 21+\end{array}$

Practice 07: Small, ${ }^{c}$ urban, university nurse-led FQHC; Not participating in NCQA $\begin{array}{llllll}\text { P07-NP1 } & 51-60 & F & N P & N & 1-5\end{array}$ $\begin{array}{llllll}\text { P07-NP2 } & 21-30 & \mathrm{~F} & \mathrm{NP} & \mathrm{N} & 1-5\end{array}$ $\begin{array}{llllll}\text { P07-NP3 } & 21-30 & F & N P & N & 1-5\end{array}$ $\begin{array}{llllll}\text { P07-NP4 } & 51-60 & F & \text { NP } & Y & 1-5\end{array}$

continues

$\mathrm{DO}=$ doctor of osteopathic medicine; $\mathrm{DPC}=$ direct primary care; $\mathrm{F}=$ female; $\mathrm{FM}=$ family medicine; $\mathrm{FQHC}=$ Federally Qualified Health Center; ID = identification; IM = internal medicine; $M=$ male; $\mathrm{MD}=$ medical doctor; NCQA = National Committee for Quality Assurance; NP = nurse practitioners;

Small $=<5$ physicians.

however, had been that "historically, oncologists are very possessive of their patients. Once they're an oncology patient, they're an oncology patient." [P06-MD1] A variation on that perspective highlighted the patient's role in this dynamic. To illustrate, one physician described a patient who had breast cancer 13 years ago with no recurrence, but continued to see her oncologist every 6 months for blood work:

I said, "Well, I can do the surveillance for you, and if anything happens, I'd be happy to refer you back to oncology immediately"... I think she's built up this trust in [the oncologist] that she is fearful to go to anybody but him... [P06-MD2] 
Table 1. Practice Descriptions and Characteristics of Clinicians Interviewed in Each (continued)

\begin{tabular}{|c|c|c|c|c|c|}
\hline $\begin{array}{l}\text { Practice and } \\
\text { Clinician ID }\end{array}$ & $\begin{array}{l}\text { Age } \\
\text { Range, Y }\end{array}$ & Sex & $\begin{array}{l}\text { Degreel } \\
\text { Specialty }\end{array}$ & $\begin{array}{l}\text { Leadership } \\
\text { Role (Y/N) }\end{array}$ & $\begin{array}{l}\text { Years at } \\
\text { Practice }\end{array}$ \\
\hline \multicolumn{6}{|c|}{ Practice 08: Small,' suburban, Independent, physician-owned; NCQA Level 3} \\
\hline P08-MD1 & $41-50$ & $\mathrm{~F}$ & $\mathrm{DO} / \mathrm{FM}$ & Y & $11-20$ \\
\hline P08-MD2 & $61-70$ & M & $\mathrm{MD} / \mathrm{FM}$ & Y & $21+$ \\
\hline \multicolumn{6}{|c|}{ Practice 09: Medium, ${ }^{b}$ urban, capitated, non-profit, independent; NCQA Level 3} \\
\hline P09-MD1 & $61-70$ & M & MD/FM & Y & $6-10$ \\
\hline \multicolumn{6}{|c|}{ Practice 10: Large, ${ }^{a}$ urban, academic hospital health system-owned; NCQA Level 3} \\
\hline P10-MD1 & $51-60$ & M & MD/IM & Y & $1-5$ \\
\hline P10-MD2 & $41-50$ & $\mathrm{M}$ & $\mathrm{MD} / \mathrm{IM}$ & $\mathrm{N}$ & $1-5$ \\
\hline P10-PA1 & $31-40$ & $\mathrm{~F}$ & $\mathrm{PA}$ & $\mathrm{N}$ & $1-5$ \\
\hline \multicolumn{6}{|c|}{ Practice 11: Medium, ${ }^{\mathrm{b}}$ suburban, VA; Not participating in NCQA } \\
\hline P11-MD1 & $51-60$ & $\mathrm{~F}$ & MD/IM & $\mathrm{N}$ & $11-20$ \\
\hline \multicolumn{6}{|c|}{$\begin{array}{l}\text { Practice 12: Medium, }{ }^{b} \text { urban, independent, physician-owned, multi-site, DPC, Not } \\
\text { participating in NCQA }\end{array}$} \\
\hline P12-MD1 & $31-40$ & M & $\mathrm{MD} / \mathrm{FM}$ & $\mathrm{N}$ & $1-5$ \\
\hline P12-MD2 & $41-50$ & $\mathrm{~F}$ & $\mathrm{MD} / \mathrm{FM}$ & Y & $6-10$ \\
\hline P12-MD3 & $41-50$ & $\mathrm{~F}$ & MD/FM & Y & $6-10$ \\
\hline P12-PA1 & $31-40$ & $\mathrm{~F}$ & PA & $\mathrm{N}$ & $1-5$ \\
\hline \multicolumn{6}{|c|}{$\begin{array}{l}\text { Practice 13: Small,' suburban, independent, Medicare Advantage; Not participating } \\
\text { in NCQA }\end{array}$} \\
\hline P13-MD1 & $41-50$ & $M$ & MD/FM & $Y$ & $1-5$ \\
\hline P13-MD2 & $51-60$ & $\mathrm{~F}$ & DO/FM & $\mathrm{N}$ & $<1$ \\
\hline P13-MD3 & $51-60$ & $M$ & MD/FM & $\mathrm{N}$ & $1-5$ \\
\hline \multicolumn{6}{|c|}{$\begin{array}{l}\text { Practice 14: Small, ‘ small city, independent, physician-owned, DPC, Not participating } \\
\text { in NCQA }\end{array}$} \\
\hline P14-MD1 & $31-40$ & $M$ & MD/FM & Y & $1-5$ \\
\hline P14-MD2 & $41-50$ & $M$ & MD/FM & Y & $1-5$ \\
\hline \multicolumn{6}{|c|}{$\begin{array}{l}\mathrm{DO}=\text { doctor of osteopathic medicine; } \mathrm{DPC}=\text { direct primary care; } \mathrm{F}=\text { female; } \mathrm{FM}=\text { family medicine; } \\
\mathrm{FQHC}=\text { Federally Qualified Health Center; } \mathrm{ID}=\text { identification; } \mathrm{IM}=\text { internal medicine; } \mathrm{M}=\text { male; } \\
\mathrm{MD}=\text { medical doctor; } \mathrm{NCQA}=\text { National Committee for Quality Assurance; } \mathrm{NP}=\text { nurse practitioners; } \\
\mathrm{PA}=\text { physician assistant; } \mathrm{VA}=\text { Veterans Administration. }\end{array}$} \\
\hline \multicolumn{6}{|c|}{${ }^{\mathrm{a}}$ Large $=>10$ physicians. } \\
\hline \multicolumn{6}{|c|}{${ }^{\mathrm{b}}$ Medium $=5-10$ physicians. } \\
\hline 'small $=<5$ physi & & & & & \\
\hline
\end{tabular}

push [our system] to do this too, because you can't just do it one patient at a time. [P03-MD3]

Another physician spoke of cancer survivors as a distinct population, but felt national recognition would be necessary to support a systematic approach to care:

[W] e haven't included cancer as one of the major chronic diseases that we have to follow. There's these big five: heart disease, diabetes, heart failure... but more and more cancer is a chronic disease for many people now... I mean, that's not recognized across the board of: what are we doing with our cancer patients? Are they getting appropriate follow-up?... Well, I suppose you have to bring it to the attention, at the national level. [P05-MD1]

Other clinicians viewed cancer survivors like all other patients with chronic diseases and not as a distinct population with unique needs. These clinicians' inability to articulate the contours of survivorship care seemed to stem from their generalist philosophy. As primary care clinicians, they saw themselves as responsible for the totality of patients' health, where cancer history was just one tile in a complex mosaic of needs. One nurse practitioner explained:

I think in our training, we're just taught to look at the person as a whole. We don't look at [just] cancer. That's a piece of you; that's not you. We're just as interested in, "Can you pay for your heat?" as we are, "Do you have rectal bleeding?" Because it all matters at the end of the day. [P07-NP4]

Some of these clinicians had trouble teasing out distinctions between their usual provision of care and their care for cancer survivors specifically. Thus, they subsumed survivorship under the broader category of whole-person care. One physician commented:

I'd be interested what all the recommendations from the [IOM] are. My bet is that a good family doc is going to be doing these things, anyway... checking for recurrence and have it on their radar at each annual check or even more frequently. I would hope a good family doc is going to be evaluating for psychosocial... problems that are happening as a result of cancer that [patients] recently or even not so recently dealt with. [P14-MD1]
I'm very well aware of it, and we don't [have a systematic approach], so it doesn't flash up on the screen; you have to remember [that the patient had cancer]... I really want to 


\section{An Identity Crisis in Primary Care}

In addition to divergent views among the clinicians interviewed, we also found a general lack of coherent knowledge about how to care for cancer survivors. Several clinicians expressed mixed opinions, contradicted themselves, vacillated on their stance, or paused when asked about their/primary care's role in cancer survivorship care. In fact, some clinicians struggled to talk about cancer survivorship at all in their interviews. As qualitative analysts, we were not expecting this amount of dissonance and incoherence, which pushed us to seek deeper understanding of these findings. Further interpretation of the data through the lens of this incoherence and diversity of opinion surfaced a broader issue of identity within the primary care field. A deeply ingrained professional focus on whole-person and relationship-centered care presents throughout the interviews. These clinicians, with an identity based on delivering whole-person, comprehensive, coordinated care, appeared to hit a wall of identity confusion when confronted with a swiftly changing highly specialized knowledge base and a highly variable group of patients referred to as "cancer survivors."

Some clinicians dealt with this confusion by limiting the boundary of their identity and excluding cancer survivorship from their scope of care and professional responsibility. Others struggled to maintain a comprehensive primary care identity but in different ways. Some wanted to include cancer survivor care, but found the knowledge gap too difficult and pleaded for help, while others shifted the focus to problematic relationships with oncologists. Another group seemed to minimize the uniqueness of cancer survivors and viewed a history of cancer as just another risk factor to manage. Yet, none of these "solutions" appeared to resolve the dissonance and incoherence.

\section{DISCUSSION}

Experts believed cancer survivorship care plans and clinical guidelines would be accepted and used in primary care practices. ${ }^{7,9-13}$ As in prior research, ${ }^{22,29}$ however, we found a lack of congruence between clinical guidelines and survivorship knowledge and practices, with no clinician we interviewed articulating best practices in cancer survivorship care as outlined in clinical guidelines. These findings tap into a deeper national discussion concerning the role and identity of primary care. ${ }^{30-32}$ Stein identified 3 family medicine identities that correspond to the different approaches to cancer survivor care discerned in our data. ${ }^{33}$ One is the holistic biopsychosocial identity, exemplified by those diminishing the uniqueness of cancer survivor care. A second is more pragmatic, consisting of traits responsive to market forces and personal preferences, which resonates with the clinicians who set boundaries excluding cancer survivor care. Stein's third identity reflects how the larger health care system defines family medicine and mirrors those interviewees frustrated by the obstacles to cancer survivor care. Thus, cancer survivorship care plans and guidelines will never be sufficient without facing the unresolved professional identity issues regarding survivorship care.

We acknowledge a number of limitations to this study, including not triangulating findings with perspectives of patients or oncologists. Future research should explore the role of patients' relationships with oncologists. Even if it makes good medical sense for primary care to be the hub of survivorship care, it may not make sense to patients who are attached to their oncologists after having gone through a lifethreatening diagnosis, or to oncologists who may cherish seeing their "successes." Another limitation is that clinician participants were all from practices with national reputations as workforce innovators. We believe this amplifies the urgency of the findings, that even among exemplars, none had established systematic strategies for cancer survivorship care, so the situation is unlikely to be better in practices that are more typical. We only conducted individual interviews, so future research might gain additional insights through focus groups that facilitate group dynamic.

Despite these limitations, it is clear that enhancing the primary care of cancer survivors requires overcoming the gap between cancer specialty guidelines and primary care clinicians' perspectives concerning cancer survivorship. While any new innovation or recommendation has a lag in reaching the front lines of clinical practice ${ }^{34}$ the wide range of primary care sensibilities around cancer survivorship care indicate that more than the usual dissemination efforts are needed. Scholars of professional identity formation have recently advocated viewing medicine as professional communities of practice to help clinicians adapt and maintain professional identity in the face of rapid change. ${ }^{35}$

Cruess and colleagues conceptualize professional identity formation at the top of a pyramid built on knowledge ("knows"), competence ("knows how"), performance ("shows how"), action ("does"), and identity $\left(\right.$ "is"). ${ }^{36}$ Barnhoon emphasizes the layered interplay of these stages, including the important additions of environment and beliefs with mission at the center, the core of being a doctor. ${ }^{37}$ We define professional identity in primary care as how one conceives of oneself as a primary care doctor and/or clinician. ${ }^{38}$ For primary care physicians, this professional identity includes both their identity as doctors and their specific identity as primary care clinicians or generalists. ${ }^{39}$ In medical 
school, students develop their "doctor" identity as they learn to be conversant in the languages of pathology, pathophysiology, and care options across the different specialties. During primary care residency, physicians form their generalist identity as they learn to translate and interpret knowledge for the functions of prioritizing, integrating, and personalizing a management plan for each person, while also considering their values, context, and particularities. The larger "doctor" identity provides a potential common ground for primary care negotiating the "contested" generalist/specialist boundaries of cancer care with oncologists.

Solutions for expanding primary care's role in caring for patients with a history of cancer must certainly include clarifying the naming, specification, and content of this care. There is some evidence, although effectiveness data are limited, that primary care physicians can provide certain care adequately to some cancer patients, including addressing psychological distress and other patient reported outcomes, ${ }^{40}$ and that some practices have the capacity to coordinate care for survivors. ${ }^{21}$ However, there is also documentation of varying knowledge gaps and ability to implement guideline-recommended care for survivors within primary care settings. For example, Luctkar-Flude and colleagues observed that while primary care physicians more routinely adhered to guidelines for surveillance and weight management for breast cancer patients, management of other long-term effects for breast cancer patients, such as fatigue, were not well implemented. ${ }^{41}$ They found despite being willing to provide survivorship care, primary care providers expressed anxiety related to gaps in knowledge and experience.

Limited cancer-care training discourages primary care clinicians and their patients from feeling competent in their ability to manage cancer-related issues. ${ }^{42-44}$ Unlike care for patients with heart disease or diabetes, care for long-term cancer survivors is currently not an integral part of medical school education or primary care residency training. ${ }^{7}$ To increase physician training in care of patients with history of cancer, family medicine and internal medicine certification boards and professional organizations need to create new educational pieces and continuing medical education (CME) content in support of cancer survivorship care. The current absence of the above means the primary care of patients with a history of cancer remains invisible during the critical early years of professional identity formation and during continuing education, and partially explains why cancer survivorship care now represents an identity crisis.

More perplexing may be overcoming the identity crisis created when primary care's deeply ingrained philosophy of whole-person and relationship-centered care $^{45-48}$ along with integrating ${ }^{48-50}$ and prioritizing ${ }^{51-55}$ functions, faces highly complicated and rapidly changing specialized knowledge. The most recent definition of the roles and identity for family physicians includes 6 core features. ${ }^{56}$ The following 4 are all challenged by insufficient training to monitor and manage potential complications of toxic and rapidly changing cancer treatments: (1) personal doctors for all ages and health conditions; (2) first contact care at which most issues are addressed ${ }_{i}(3)$ enduring partnerships with patients facilitating prevention, understanding, management, navigation, and goal setting; and (4) adapting care to the unique needs of patients and communities. Furthermore, the rising number of patients with multimorbidity, ${ }^{57.59}$ concerns about the diminishing scope of care of primary care clinicians, ${ }^{31,32}$ and the growth in number of advanced practice clinicians delivering primary care also challenge primary care physicians' professional identity. ${ }^{60-62}$ The accelerated development of specialized technologies, pharmaceuticals, diagnostics, and knowledge in genetics, advanced cardiovascular, neurologic, and other specialty care represent knowledge challenges similar to cancer survivorship care.

How do we preserve the comprehensive and integrative functions of primary care while overcoming the apparent identity crisis? As tasks and complexity increase, those functions and their supporting professional identity are tested. One option is to shed scope of care and construct boundaries of management, continuity, and relationship and even create new silos for care of patients with "specialized" needs such as cancer survivors. ${ }^{63}$ This will likely accelerate the current problematic fragmentation of care. Another option is to enhance the tools for translation of new knowledge and focus residency and post-residency training more on the craft functions and less on specialized content. Primary care, along with oncology and other specialties, might consider coproducing and translating new knowledge about care for cancer survivors that primary care clinicians can prioritize, personalize, and integrate to address patients' needs and values within a shared decision-making framework. It is not only the care of cancer survivors that is at stake as primary care struggles to embody its professional identity around its mission to deliver personal generalist care in service to patients and their communities. As noted by Hafferty and colleagues, "We see medicine's future as a profession being defined within an ongoing (and necessary) tension between the siren calls of system controls and bureaucratic stability, and a more collective sense of physicianhood as a disruptive and quasi-subversive work force-in the service of patients." ${ }^{164} \mathrm{New}$ knowledge-translation transfer platforms for generalists, along with new curricula for medical schools and 
residencies on how to do this, and developing medicine as a community of practice will be required.

To read or post commentaries in response to this article, see it online at http://www.AnnFamMed.org/content/18/3/202.

Key words: primary care; professional identity; cancer survivors; patient-centered medical home; qualitative research

Submitted February 26, 2019; submitted, revised, June 27, 2019; accepted August 13, 2019.

Funding support: Funding for this research was provided by National Cancer Institute Grant R01 CA176545, "PCMH Implementation Strategies: Implications for Cancer Survivor Care" (PI: Crabtree). Dr Stange's time is supported in part by a Clinical Research Professorship from the American Cancer Society.

Previous presentation: Oral presentation at the 2018 NAPCRG International Conference; December 10-11, 2018; Tampa, Florida.

Acknowledgments: The authors wish to thank Kevin Oeffinger, MD; Paul Nutting, MD; Carlos Jaen, MD, PhD; and Larissa Nekhlyudov, MD, who served as members of the executive steering committee and provided input on our study design and sampling framework.

Ethical approval: This study was approved by the Rutgers Biomedical and Health Sciences Institutional Review Board (IRB Protocol No: 2013003629).

\section{References}

1. Bluethmann SM, Mariotto $A B$, Rowland JH. Anticipating the "silver tsunami": prevalence trajectories and comorbidity burden among older cancer survivors in the United States. Cancer Epidemiol Biomarkers Prev. 2016;25(7):1029-1036.

2. Phillips SM, Padgett LS, Leisenring WM, et al. Survivors of childhood cancer in the United States: prevalence and burden of morbidity. Cancer Epidemiol Biomarkers Prev. 2015;24(4):653-663.

3. Debono D. Coping with the oncology workforce shortage: transitioning oncology follow-up care to primary care providers. J Oncol Pract. 2010;6(4):203-205.

4. Erikson C, Salsberg E, Forte G, Bruinooge S, Goldstein M. Future supply and demand for oncologists : challenges to assuring access to oncology services. J Oncol Pract. 2007;3(2):79-86.

5. Erikson C, Schulman S, Kosty M, Hanley A. Oncology workforce: results of the ASCO 2007 program directors survey. J Oncol Pract. 2009;5(2):62-65.

6. Association of American Medical Colleges. The Complexities of Physician Supply and Demand: Projections from 2013 to 2025. Washington, DC: IHS Inc; 2015.

7. Hewitt ME, Greenfield S, Stovall E, National Cancer Policy Board (US). Committee on Cancer Survivorship: Improving Care and Quality of Life. From cancer patient to cancer survivor: lost in transition. Washington, DC: National Academies Press; 2006.

8. Rubin G, Berendsen A, Crawford SM, et al. The expanding role of primary care in cancer control. Lancet Oncol. 2015;16(12):1231-1272.

9. Cohen EE, LaMonte SJ, Erb NL, et al. American Cancer Society head and neck cancer survivorship care guideline. CA Cancer J Clin. 2016; 66(3):203-239.

10. El-Shami K, Oeffinger KC, Erb NL, et al. American Cancer Society colorectal cancer survivorship care guidelines. CA Cancer J Clin. 2015;65(6):428-455.
11. Runowicz CD, Leach CR, Henry NL, et al. American Cancer Society/ American Society of Clinical Oncology breast cancer survivorship care guideline. CA Cancer J Clin. 2016;66(1):43-73.

12. Skolarus TA, Wolf AM, Erb NL, et al. American Cancer Society prostate cancer survivorship care guidelines. CA Cancer J Clin. 2014; 64(4):225-249.

13. Earle CC. Failing to plan is planning to fail: improving the quality of care with survivorship care plans. J Clin Oncol. 2006;24(32): 5112-5116.

14. Nekhlyudov L, O'malley DM, Hudson SV. Integrating primary care providers in the care of cancer survivors: gaps in evidence and future opportunities. Lancet Oncol. 2017;18(1):e30-e38.

15. Feuerstein M. Defining cancer survivorship. J Cancer Surviv. 2007; 1(1):5-7.

16. Khan NF, Rose PW, Evans J. Defining cancer survivorship: a more transparent approach is needed. J Cancer Surviv. 2012;6(1):33-36.

17. Mayer DK, Nasso SF, Earp JA. Defining cancer survivors, their needs, and perspectives on survivorship health care in the USA. Lancet Oncol. 2017;18(1):e11-e18.

18. National Cancer Institute. NCI Dictionary of Cancer Terms. https:// www.cancer.gov/publications/dictionaries/cancer-terms/def/survivor. Accessed Dec 2, 2018.

19. Jacobsen PB, Nipp RD, Ganz PA. Addressing the survivorship care needs of patients receiving extended cancer treatment. Am Soc Clin Oncol Educ Book. 2017;37:674-683.

20. Rubinstein EB, Miller WL, Hudson SV, et al. Cancer survivorship care in advanced primary care practices: a qualitative study of challenges and opportunities. JAMA Intern Med. 2017;177(12):1726-1732.

21. Tsui J, Hudson SV, Rubinstein EB, et al. A mixed-methods analysis of the capacity of the patient-centered medical home to implement care coordination services for cancer survivors. Transl Behav Med. 2018;8(3):319-327.

22. Lawrence RA, McLoone JK, Wakefield CE, Cohn RJ. Primary care physicians' perspectives of their role in cancer care: a systematic review. J Gen Intern Med. 2016;31(10):1222-1236.

23. Crabtree BF, Nutting PA, Miller WL, et al. Primary care practice transformation is hard work: insights from a 15-year developmental program of research. Med Care. 2011;49(Suppl):S28-S35.

24. Crabtree BF, Nutting PA, Miller WL, Stange KC, Stewart EE, Jaen CR. Summary of the National Demonstration Project and recommendations for the patient-centered medical home. Ann Fam Med. 2010;8 Suppl 1:S80-90; S92.

25. Miller WL, Crabtree BF, Nutting PA, Stange KC, Jaen CR. Primary care practice development: a relationship-centered approach. Ann Fam Med. 2010;8 Suppl 1:S68-79; S92.

26. Nutting PA, Crabtree BF, Miller WL, Stange KC, Stewart E, Jaén C. Transforming physician practices to patient-centered medical homes: lessons from the national demonstration project. Health Aff (Millwood). 2011;30(3):439-445.

27. Wagner EH, Flinter M, Hsu C, et al. Effective team-based primary care: observations from innovative practices. BMC Fam Pract. 2017; 18(1):13.

28. Miller WL, Crabtree BF. The Dance of Interpretation. In: Crabtree BF, Miller WL, eds. Doing Qualitative Research. 2nd ed. Thousand Oaks, CA: Sage Publications; 1999:127-143.

29. Potosky AL, Han PK, Rowland J, et al. Differences between primary care physicians' and oncologists' knowledge, attitudes and practices regarding the care of cancer survivors. J Gen Intern Med. 2011; 26(12):1403-1410.

30. Coutinho AJ, Cochrane A, Stelter K, Phillips RL Jr, Peterson LE. Comparison of intended scope of practice for family medicine residents with reported scope of practice among practicing family physicians. JAMA. 2015;314(22):2364-2372. 
31. Grumbach K. To be or not to be comprehensive. Ann Fam Med. 2015;13(3):204-205.

32. Saultz J. Comprehensive practice. Fam Med. 2017;49(2):89-90.

33. Stein HF. Family medicine's identity: being generalists in a specialist culture? Ann Fam Med. 2006;4(5):455-459.

34. Westfall JM, Mold J, Fagnan L. Practice-based research-"Blue Highways" on the NIH roadmap. JAMA. 2007;297(4):403-406.

35. Cruess RL, Cruess SR, Steinert Y. Medicine as a community of practice: implications for medical education. Acad Med. 2018;93(2):185-191.

36. Cruess RL, Cruess SR, Steinert Y. Amending Miller's Pyramid to include professional identity formation. Acad Med. 2016;91(2):180-185.

37. Barnhoorn PC. Professional identity formation: onions rather than pyramids. Acad Med. 2016;91(3):291.

38. Wilson I, Cowin LS, Johnson M, Young H. Professional identity in medical students: pedagogical challenges to medical education. Teach Learn Med. 2013;25(4):369-373.

39. Carney PA, Waller E, Eiff MP, et al. Measuring family physician identity: the development of a new instrument. Fam Med. 2013; 45(10):708-718.

40. Grunfeld E, Julian JA, Pond G, et al. Evaluating survivorship care plans: results of a randomized, clinical trial of patients with breast cancer. J Clin Oncol. 2011;29(36):4755-4762.

41. Luctkar-Flude M, Aiken A, McColl MA, Tranmer J, Langley H. Are primary care providers implementing evidence-based care for breast cancer survivors? Can Fam Physician. 2015;61(11):978-984.

42. Bell K. The breast-cancer-ization of cancer survivorship: implications for experiences of the disease. Soc Sci Med. 2014;110:56-63.

43. Mao JJ, Bowman MA, Stricker CT, et al. Delivery of survivorship care by primary care physicians: the perspective of breast cancer patients. J Clin Oncol. 2009;27(6):933-938.

44. Schütze $H$, Chin M, Weller D, Harris MF. Patient, general practitioner and oncologist views regarding long-term cancer shared care. Fam Pract. 2018;35(3):323-329.

45. Beach MC, Inui T; Relationship-Centered Care Research Network. Relationship-centered care: a constructive reframing. J Gen Intern Med. 2006;21(Suppl 1):S3-S8.

46. Epstein RM, Fiscella K, Lesser CS, Stange KC. Why the nation needs a policy push on patient-centered health care. Health Aff (Millwood). 2010;29(8):1489-1495.

47. Safran DG, Miller W, Beckman H. Organizational dimensions of relationship-centered care. Theory, evidence, and practice. J Gen Intern Med. 2006;21(Suppl 1):S9-S15.

48. Stange KC. The generalist approach. Ann Fam Med. 2009;7(3):198-203.

49. Bayliss EA, Bonds DE, Boyd CM, et al. Understanding the context of health for persons with multiple chronic conditions: moving from what is the matter to what matters. Ann Fam Med. 2014;12(3): 260-269.
50. Stange KC. The problem of fragmentation and the need for integrative solutions. Ann Fam Med. 2009;7(2):100-103.

51. Kamerow DB. Prioritizing prevention. J Fam Pract. 1994;38(3) 229-230.

52. O'Connor PJ, Sperl-Hillen JM, Margolis KL, Kottke TE. Strategies to prioritize clinical options in primary care. Ann Fam Med. 2017;15(1): 10-13.

53. Stange KC. A science of connectedness. Ann Fam Med. 2009;7(5): 387-395.

54. Stange KC. Ways of knowing, learning, and developing. Ann Fam Med. 2010;8(1):4-10.

55. Stange KC, Etz RS, Gullett $H$, et al. Metrics for assessing improvements in primary health care. Annu Rev Public Health. 2014;35: 423-442.

56. Phillips RL Jr, Brundgardt S, Lesko SE, et al. The future role of the family physician in the United States: a rigorous exercise in definition. Ann Fam Med. 2014;12(3):250-255.

57. Cassell A, Edwards D, Harshfield A, et al. The epidemiology of multimorbidity in primary care: a retrospective cohort study. $\mathrm{Br} / \mathrm{Gen}$ Pract. 2018;68(669):e245-e251.

58. King DE, Xiang J, Pilkerton CS. Multimorbidity trends in United States adults, 1988-2014. J Am Board Fam Med. 2018;31(4):503-513.

59. Roberts KC, Rao DP, Bennett TL, Loukine L, Jayaraman GC. Prevalence and patterns of chronic disease multimorbidity and associated determinants in Canada. Health Promot Chronic Dis Prev Can. 2015; 35(6):87-94.

60. Auerbach DI, Chen PG, Friedberg MW, et al. Nurse-managed health centers and patient-centered medical homes could mitigate expected primary care physician shortage. Health Aff (Millwood). 2013:32(11):1933-1941.

61. Auerbach DI, Staiger DO, Buerhaus PI. Growing ranks of advanced practice clinicians - implications for the physician workforce. $N$ Engl J Med. 2018;378(25):2358-2360.

62. Hooker RS, McCaig LF. Use of physician assistants and nurse practitioners in primary care, 1995-1999. Health Aff (Millwood). 2001; 20(4):231-238.

63. Porter ME, Pabo EA, Lee TH. Redesigning primary care: a strategic vision to improve value by organizing around patients' needs. Health Aff (Millwood). 2013;32(3):516-525.

64. Hafferty FW, Michalec B, Martimianakis MA, Tilburt JC. alternative framings, countervailing visions: locating the " $\mathrm{P}$ " in professional identity formation. Acad Med. 2016;91(2):171-174. 